\title{
Dark-State Luminescence of Macroatoms at the Near Field
}

\author{
Ulrich Hohenester, ${ }^{1 *}$ Guido Goldoni, ${ }^{2}$ and Elisa Molinari ${ }^{2}$ \\ ${ }^{1}$ Institut für Physik, Karl-Franzens-Universität Graz, Universitätsplatz 5, 8010 Graz, Austria \\ ${ }^{2} I N F M-S^{3}$ and Dipartimento di Fisica, Università di Modena e Reggio Emilia, Via Campi 213/A, 41100 Modena, Italy
}

(Received 8 July 2005; published 16 November 2005)

\begin{abstract}
We theoretically analyze the optical near-field response of a semiconductor macroatom induced by local monolayer fluctuations in the thickness of a semiconductor quantum well, where the large active volume results in a strong enhancement of the light-matter coupling. We find that in the near-field regime bright and dark excitonic states become mixed, opening new channels for the coupling to the electromagnetic field. As a consequence, ultranarrow luminescence lines appear in the simulated two-photon experiments, corresponding to very long lived excitonic states, which undergo Stark shift and Rabi splitting at relatively small field intensities.
\end{abstract}

PACS numbers: 73.21.La, 71.35. $-\mathrm{y}, 78.67 .-\mathrm{n}$

Scanning near-field optical microscopy (SNOM) is an experimental technique that combines the advantages of nanometric resolution of scanning-probe microscopy with the unique properties of optical spectroscopy. This is achieved by quenching light through the tip of an optical fiber and exciting the probe in the near field, thereby overcoming the diffraction limit of light by several orders of magnitude [1,2]. Near-field spectroscopy has been successfully used for the measurement of single and coupled semiconductor nanostructures [3-5], molecules [6,7], or metallic nanostructures $[8,9]$. If the spatial near-field resolution falls below the extension of confined quantum systems, it becomes possible to directly map out the spatial probability distribution of the wave function. This was recently achieved by Matsuda et al. [10] for quantum dots where the quantum confinement is induced by local monolayer fluctuations in the thickness of a semiconductor quantum well. In the future, similar wave function mapping is expected to become possible for other scanningprobe techniques, such as optical nanoantennas [11,12], which might pave the way for optical nanoimaging of molecules or biological structures.

In contrast to the far field, where the matrix elements governing the light-matter coupling are determined by the quantum states alone, in the near field the pertinent matrix elements become a convolution of the quantum states with the electromagnetic field profile of the SNOM tip [13]. This allows one to break in the near field the usual optical selection rules and to excite dark states whose excitation is forbidden by symmetry in the far field. Spatial maps of dark states in semiconductor nanostructures were simulated for high-resolution SNOM in absorption [14,15] and luminescence [16,17], and were shown to depend sensitively on the spatial near-field resolution. This previous work focused on the analysis of the near-field matrix elements, while assuming for simplicity that dark states couple to their environment in the same manner as bright states do. However, because of the reduced interaction channels of dark states with their environment the dephasing and relaxation dynamics are expected to be different with respect to their bright counterparts, and to be further influenced by the presence of the exciting near-field probe, as, e.g., recently reported for a single quantum dot in close proximity to a scanning optical antenna [12]. Also in the field of the celebrated $1 S-2 S$ high-resolution laser spectroscopy of the hydrogen atom, such modification of environment couplings has been shown to have a decisive influence on the spectral properties of dark states [18]: the optically forbidden $2 S$ state, which is excited in a two-photon process, is extremely long lived and depends sensitively on the excitation and environment conditions.

In this Letter we theoretically investigate the spectral response of a semiconductor quantum dot excited in the optical near field. We find that in order to observe dark states directly by means of optical spectroscopy it not only suffices to excite these states, e.g., by means of symmetry breaking in the near field, but additionally new decay channels have to be opened that allow coupling to propagating photon modes. A unique system for such investigation is provided by macroatoms induced by local monolayer fluctuations in the thickness of a semiconductor quantum well, where the large active volume results in a strong enhancement of the light-matter coupling. This strong coupling combines with the dot extensions larger than the resolution of the electromagnetic near-field probe in opening new decay channels, which allow one to optically access very long lived dark states. We predict that, as a consequence, ultranarrow luminescence lines appear in two-photon experiments, not present in the farfield regime. Such lines undergo Stark shift and Rabi splitting at relatively small field intensities. Optical nearfield spectroscopy of dark states could allow the measurement of weak scatterings in solids, and might provide an excellent laboratory to address general questions regarding local spectroscopies and quantum measurements in nanosystems.

In this work we consider quantum confined states induced by monolayer fluctuations in the thickness of a GaAs/AlGaAs semiconductor quantum well [Fig. 1(a)] $[10,14]$. Since the confinement length (several tens of 
a
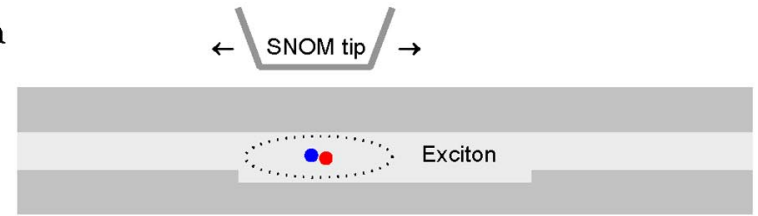

b

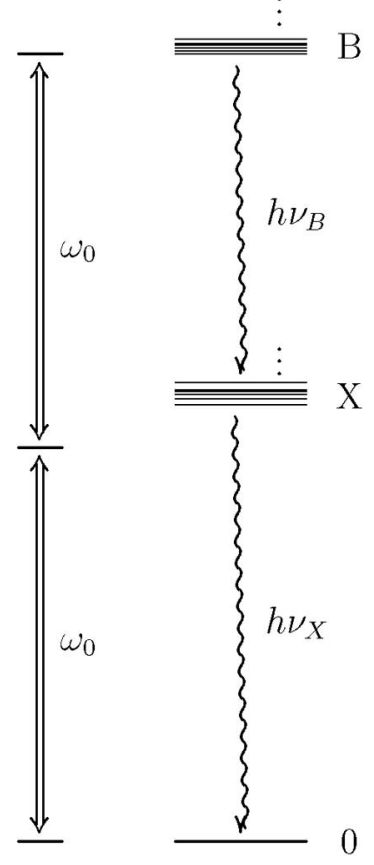

$|\Psi(R)|^{2}$
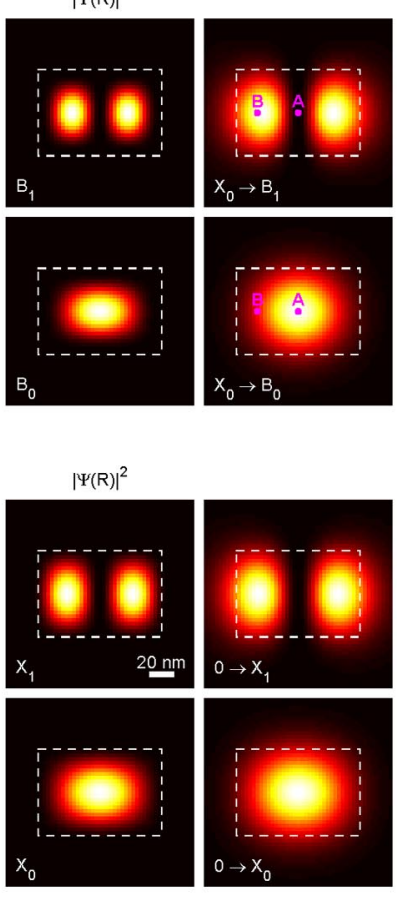

FIG. 1 (color online). (a) Schematic sketch of the confinement potential for excitons and biexcitons, which is induced by local monolayer fluctuations in the thickness of a semiconductor quantum well, and the probing SNOM tip. (b) Photoexcitation scenario assumed in our calculations. A biexciton state is excited by SNOM within a two-photon process. Because of the biexciton binding $\delta E_{b} \sim 4 \mathrm{meV}$, the luminescence of the biexciton-toexciton transition is redshifted and the exciton-to-ground-state transition blueshifted with respect to $\omega_{0}$. The insets report the square modulus of the center-of-mass part of the exciton and biexciton wave functions [14,19] (left columns) and the spatial near-field maps (right columns) for a terrace of dimension $100 \mathrm{~nm} \times 70 \mathrm{~nm}$ (dashed line) and for a Bethe-Bouwkamp near-field probe with a full width of half maximum of approximately $25 \mathrm{~nm}$. In the optical far field only $X_{0}$ and $B_{0}$ are allowed, whereas $X_{1}$ and $B_{1}$ are forbidden because of symmetry.

$\mathrm{nm}$ ) is much larger than both the lattice constant and the excitonic Bohr radius, we adopt the usual envelope function and rigid-exciton (rigid-biexciton) approximations, the latter assuming that the correlated electron-hole wave functions factorize into a center of mass and a relative part given by that of the quantum well $[14,19,20]$. The insets of Fig. 1(b), left column, show the real-space maps of the square modulus of the exciton and biexciton ground $\left(X_{0}, B_{0}\right)$ and first excited $\left(X_{1}, B_{1}\right)$ states, respectively, for a prototypical squarelike confinement potential (dashed lines) as a function of the center-of-mass coordinates.

The wave function symmetries resemble those of a particle-in-a-box problem with $s$ and $p$ symmetry states.

The optical matrix elements for the vacuum-to-exciton and exciton-to-biexciton transitions $M_{0 x}(\boldsymbol{r})$ and $M_{x b}(\boldsymbol{r})$ can be computed according to $[14,19]$

$$
\begin{aligned}
& M_{0 x}(\boldsymbol{r})=\Psi_{x}(\boldsymbol{r}, \boldsymbol{r}), \\
& M_{x b}(\boldsymbol{r})=\int d \boldsymbol{r}_{e} d \boldsymbol{r}_{h} \Psi_{x}^{*}\left(\boldsymbol{r}_{e}, \boldsymbol{r}_{h}\right) \Psi_{b}\left(\boldsymbol{r}, \boldsymbol{r}, \boldsymbol{r}_{e}, \boldsymbol{r}_{h}\right),
\end{aligned}
$$

where $\Psi_{x}$ and $\Psi_{b}$ are the exciton and biexciton wave functions, respectively. Here we have accounted for the fact that the photogenerated electron and hole are created at the same position $\boldsymbol{r}$, and in $M_{x b}(\boldsymbol{r})$ the positions of the additional electron and hole remain at the same position $\boldsymbol{r}_{e}$ and $\boldsymbol{r}_{h}$ (note that the spin orientations of the two electronhole pairs, which we do not indicate explicitly, are antiparallel). From Eq. (1) we obtain the usual far-field matrix elements $\bar{M}_{0 x}$ and $\bar{M}_{x b}$ by integrating over all positions $\boldsymbol{r}$. One can also obtain spatial near-field absorption maps of the quantum states by convoluting $M_{0 x}(\boldsymbol{r})$ and $M_{x b}(\boldsymbol{r})$ with the electromagnetic field profile generated by the SNOM tip. In the insets of Fig. 1(b), right column, we show such maps for the $0 \rightarrow X$ and $X \rightarrow B$ transitions [14], respectively, using a Bethe-Bouwkamp near-field distribution [1] with a spatial resolution $\sim 25 \mathrm{~nm}$. Note that in the far field only states $X_{0}$ and $B_{0}$ are optically accessible, while $X_{1}$ and $B_{1}$ are forbidden by symmetry. In the near field, instead, the SNOM tip breaks the symmetry and allows light to be absorbed also in the dark states [13-15].

We now turn to the situation where the dot is excited through the SNOM tip and relaxes through excitonic luminescence. For convenience, we consider a two-photon excitation of biexcitons [Fig. 1(b)], where, because of the biexciton binding energy $\delta E_{b} \sim 4 \mathrm{meV}$ [21], the luminescence from the $B \rightarrow X$ and $X \rightarrow 0$ recombinations is redand blueshifted, respectively, and can thus be spectrally discriminated. Photoluminescence excitation (PLE) spectroscopy, where a dark exciton state is resonantly excited, will be discussed at the end. From the convolution of the matrix elements (1) with the near-field distribution, we obtain the tip-dependent Rabi energies $\Omega_{i j}$, where $i$ and $j$ label the different states (vacuum and exciton and biexciton states) under consideration. For the incoherent dynamics, we consider phonon (acoustic deformation potential [22]) and photon scatterings. We assume that the radiative decay of excitons and biexcitons is not drastically altered by the presence of the SNOM tip. This is a reasonable assumption since the photons can be emitted into any solidangle direction, and the slightly modified photon density of states in presence of the SNOM tip is not expected to be of great importance. The Hamiltonian accounting for the exciton- and biexciton-photon couplings is then of the form $H_{o p}=\sum_{i j} g_{k}\left(a_{k}^{\dagger} \bar{M}_{i j}|i\rangle\left\langle j\left|+a_{k} \bar{M}_{i j}^{*}\right| j\right\rangle\langle i|\right)$, with $a_{k}$ the bosonic field operators for photons, $k$ the photon wave number, and $g_{k}$ the usual light-matter coupling of the bulk semiconductor $[19,23]$. 
We stress that for the system under study the splitting between the different exciton and biexciton states is comparable to the level broadenings and the Rabi energies of the driving field. In this regime, initial and final states of the recombination should be considered as mixed states renormalized by the electromagnetic interaction, which no longer posses the symmetry of the unperturbed states so that photon scatterings can occur between them. In other words, exciton and biexciton states cannot be decoupled from the light fields, and the whole system has to be treated self-consistently. A convenient approach to account for such renormalization effects is to describe photon emission processes within lowest order time-dependent perturbation theory and to employ the white-noise approximation $[23,24] \sum_{k}\left(g_{k}\right)^{2}\left\langle a_{k}(t) a_{k}^{\dagger}\left(t^{\prime}\right)\right\rangle \simeq\left(\Gamma_{\gamma} / 2\right) \delta\left(t-t^{\prime}\right)$, with $\Gamma_{\gamma}$ the Wigner-Weisskopf decay rate. This approach, which has been successfully used in quantum optics for the description of resonance luminescence [24], rests on the assumption that the photon density of states can be approximately set constant for all transitions - a good approximation because of the small exciton and biexciton level splittings in comparison to the photon energies. The time evolution of the system under consideration is then described by the master equation [19]

$$
\begin{aligned}
\dot{\rho}= & -i\left[\Delta+\Omega+\Omega^{\dagger}, \rho\right]-\frac{\Gamma_{\gamma}}{2}\left(\bar{M} \bar{M}^{\dagger} \rho+\rho \bar{M} \bar{M}^{\dagger}\right. \\
& \left.-2 \bar{M}^{\dagger} \rho \bar{M}\right)-\sum_{i}\left(L_{i}^{\dagger} L_{i} \rho+\rho L_{i}^{\dagger} L_{i}-2 L_{i} \rho L_{i}^{\dagger}\right),
\end{aligned}
$$

with $\bar{M}=\sum_{i j} \bar{M}_{i j}|i\rangle\langle j|$ and $L_{i}$ the Lindblad operators accounting for phonon scatterings. In deriving Eq. (2) we have introduced an interaction representation according to $\omega_{0} \sum_{x}|x\rangle\left\langle x\left|+2 \omega_{0} \sum_{b}\right| b\right\rangle\langle b|$, with $\Delta_{x}=E_{x}-\omega_{0}$ and $\Delta_{b}=E_{b}-2 \omega_{0}$ the detunings with respect to the driving frequency $\omega_{0}$ of the external laser. The steady-state solutions and luminescence spectra are finally obtained from Eq. (2) by computing the eigenvalues and eigenvectors of the Liouvillian and making use of the quantum regression theorem [24].

We now assume that the heterostructure is excited through a strongly inhomogeneous field emitted by a fiber tip at the two different spots A and B shown in Fig. 1(b). We also assume that the luminescence is detected somewhere in the far field (not necessarily through the SNOM tip). Figure 2 shows the corresponding luminescence spectra. For tip position A (center of the dot) the field does not break the symmetry of the underlying quantum states. Accordingly, only the ground state biexciton $B_{0}$ can be excited, whereas the transition to $B_{1}$ is forbidden because of symmetry, as in the far-field case. Correspondingly, the luminescence spectra consist of two equally strong peaks associated with the $B_{0} \rightarrow X_{0}$ and $X_{0} \rightarrow 0$ transitions. The line broadenings are due to photon emissions and are as large as $\Gamma \sim 100 \mu \mathrm{eV}$ for the large coherence volumes of the exciton and biexciton states. Note that the far-field
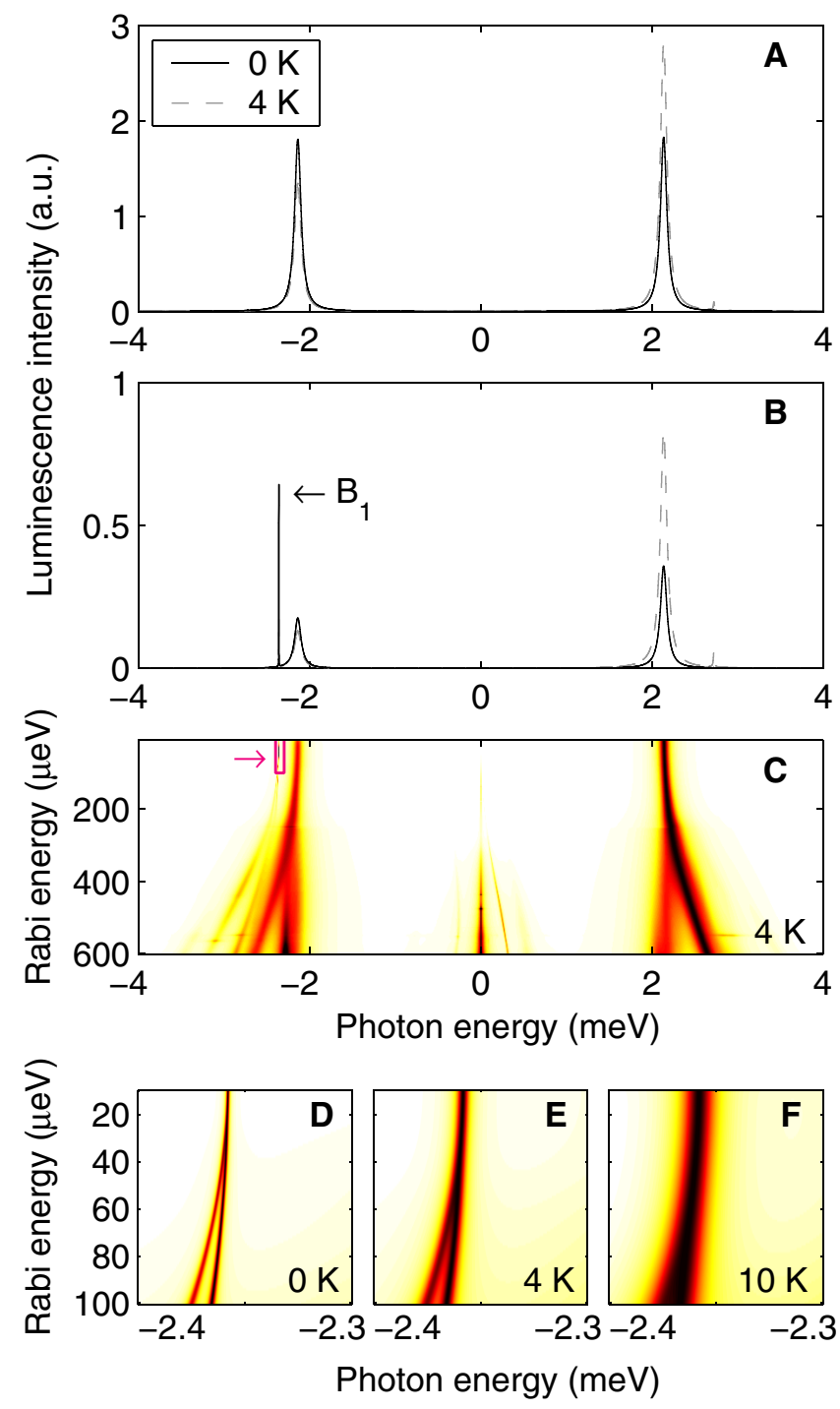

FIG. 2 (color online). Near-field luminescence spectra for two selected tip positions (a) A and (b) B, as indicated in Fig. 1(b), and different temperatures. At position $A$ the usual far-field selection rules apply, and the spectrum consists of two peaks associated with the $B_{0} \rightarrow X_{0}$ and $X_{0} \rightarrow 0$ transitions. At position $\mathrm{B}$ an additional ultranarrow peak appears, which is due to the radiative decay of the dark biexciton state $B_{1}$. Panel (c) shows the power dependence of the optical spectra at tip position $\mathrm{B}$ which have been normalized with respect to the respective maxima. The power and temperature dependence of the dark-state transition $B_{1}$ is magnified in panels (d)-(f). Photon energy zero is given by $\omega_{0}$. In our calculations we use the 12 exciton and 6 biexciton states of lowest energy.

luminescence spectrum (not shown here) is almost identical to that of Fig. 2(a).

When the dot is excited at tip position B, which does not coincide with the symmetry center of the heterostructure, an additional ultranarrow peak appears, indicated with $B_{1}$ in Fig. 2(b), which is due to the excitation of the biexciton $B_{1}$. Because this dark state couples weakly to photons, its lifetime is orders of magnitude larger than that of the bright $B_{0}$ state. At the lowest excitation powers the broadening of 
this additional peak is dominated by phonon scattering ( $\Gamma \sim 1 \mu \mathrm{eV}$ for zero temperature). However, its appearance in the luminescence spectrum indicates a substantial excitation-induced mixing of excitonic states, as also evidenced by the approximately 1:9 ratio between the integrated peak areas of the dark and bright state transitions. With increasing excitation power this mixing becomes stronger, resulting in a further peak broadening and the appearance of new emission lines as shown in panel (c). In the following we concentrate on the low power regime, corresponding to the spectra shown in Figs. 2(a) and 2(b), where the dominant mixing is between the bright $B_{0}$ and dark $B_{1}$ biexciton state, whereas exciton states become less mixed because of the off-resonant excitation and the larger level splittings due to the lighter exciton mass [14]. The power and temperature dependence of the dark-state transition $B_{1}$ is magnified in panels (d)-(f). We observe an acStark effect and a splitting of the line at relatively small Rabi energies $\Omega$. Similarly to the Mollow spectrum of a driven two-level system [24], this splitting is due to a strong light-biexciton coupling and occurs when the line broadening $\Gamma$ becomes comparable to the effective Rabi frequency (note that the $B_{1}$ transition is excited nonresonantly). With increasing temperature, $\Gamma$ increases and the Rabi splitting occurs at higher field strengths $\Omega$ [Figs. 2(e) and 2(f)]. The essentials of these findings are expected to prevail for dot confinements with lower symmetry.

An alternative to the two-photon excitation considered in this Letter is to use PLE spectroscopy to directly excite a dark exciton, which, after phonon relaxation, can be detected through the decay of the ground state exciton. Our results for such an excitation scenario show that the darkstate absorption is again spectrally extremely narrow and dominated by the Rabi energy $\Omega$.

In conclusion, we have studied luminescence from quantum dots excited through a SNOM tip. The far-field selection rules are broken and it becomes possible to excite dark biexcitons, weakly coupled to the light, which show up as additional ultranarrow peaks in the luminescence spectra. Their spectral properties depend critically on the excitation power and the sample temperature. In particular, Rabi splitting occurs for this very long lived states. Dark-state transitions in the near-field spectra are expected to constitute a genuine property of carrier complexes in large nanosystems, where the large active dot volume results in a strong enhancement of the light-matter coupling, relatively short lifetimes of bright states, and a pronounced mixing of excitonic states in presence of laser excitation (see also Ref. [25] for a related effect).

We thank Andrea Bertoni and Filippo Troiani for helpful discussions. This work has been supported in part by the EU under the TMR network "Exciting" and the Austrian science fund FWF under Project No. P15752N08. We acknowledge support from the Italian Ministry of Foreign Affairs (DGPCC), Italian Ministry of Research (FIRB-RBAU01ZEML and COFIN-2003020984), and the CINECA-INFM Supercomputing Project 2005.
*Electronic address: ulrich.hohenester@uni-graz.at

[1] M. A. Paesler and P. J. Moyer, Near-Field Optics: Theory, Instrumentation, and Applications (Wiley, New York, 1996).

[2] B. Hecht, B. Sick, U.P. Wild, V. Deckert, R. Zenobi, O. J.F. Martin, and D.W. Pohl, J. Chem. Phys. 112, 7761 (2000).

[3] E. Betzig, J. K. Trautman, T. D. Harris, J. S. Weiner, and R. L. Kostelak, Science 251, 1468 (1991).

[4] H. F. Hess, E. Betzig, T. D. Harris, L. N. Pfeiffer, and K. W. West, Science 264, 1740 (1994).

[5] J. R. Guest, T. H. Stievater, Gang Chen, E. A. Tabak, B. G. Orr, D. G. Steel, D. Gammon, and D. S. Katzer, Science 293, 2224 (2001).

[6] C. Hettich, C. Schmitt, J. Zitzmann, S. Kühn, I. Gerhardt, and V. Sandoghdar, Science 298, 385 (2002).

[7] W. E. Moerner, T. Plakhotnik, T. Irngartinger, U. P. Wild, D. W. Pohl, and B. Hecht, Phys. Rev. Lett. 73, 2764 (1994).

[8] C. Chicanne, T. David, R. Quidant, J.C. Weber, Y. Lacroute, E. Bourillot, A. Dereux, C. Golas de Francs, and C. Girard, Phys. Rev. Lett. 88, 097402 (2002).

[9] C. Ropers, D. J. Park, G. Stibenz, G. Steinmeyer, J. Kim, D. S. Kim, and C. Lienau, Phys. Rev. Lett. 94, 113901 (2005).

[10] K. Matsuda, T. Saiki, S. Nomura, M. Mihara, Y. Aoyagi, S. Nair, and T. Takagahara, Phys. Rev. Lett. 91, 177401 (2003).

[11] P. Mühlschlegel, H. J. Eisler, O. J. F. Martin, B. Hecht, and D. W. Pohl, Science 308, 1607 (2005).

[12] J. N. Farahani, D. W. Pohl, H. J. Eisler, and B. Hecht, Phys. Rev. Lett. 95, 017402 (2005).

[13] O. Mauritz, G. Goldoni, F. Rossi, and E. Molinari, Phys. Rev. Lett. 82, 847 (1999).

[14] U. Hohenester, G. Goldoni, and E. Molinari, Appl. Phys. Lett. 84, 3963 (2004).

[15] C. Simserides, U. Hohenester, G. Goldoni, and E. Molinari, Phys. Rev. B 62, 13657 (2000).

[16] S. Savasta, O. Di Stefano, and R. Girlanda, Phys. Rev. A 65, 043801 (2002).

[17] G. Pistone, S. Savasta, O. Di Stefano, and R. Girlanda, Appl. Phys. Lett. 84, 2971 (2004).

[18] The Hydrogen Atom, edited by G. F. Bassani, M. Inguscio, and T. W. Hänsch (Springer, Berlin, 1989).

[19] U. Hohenester, Handbook of Theoretical and Computational Nanotechnology, edited by M. Rieth and W. Schommers (American Scientific Publishers, Stevenson Ranch, CA, 2005).

[20] R. Zimmermann, F. Große, and E. Runge, Pure Appl. Chem. 69, 1179 (1997).

[21] A. Zrenner, L. V. Butov, M. Hagn, G. Abstreiter, G. Böhm, and G. Weimann, Phys. Rev. Lett. 72, 3382 (1994).

[22] U. Bockelmann, Phys. Rev. B 48, 17637 (1993).

[23] U. Hohenester, C. Sifel, and P. Koskinen, Phys. Rev. B 68, 245304 (2003).

[24] L. Mandel and E. Wolf, Optical Coherence and Quantum Optics (Cambridge University Press, Cambridge, 1995).

[25] A. Syouji, B.P. Zhang, Y. Segawa, J. Kishimoto, H. Ishihara, and K. Cho, Phys. Rev. Lett. 92, 257401 (2004). 